\title{
Neuromuscular control of curved walking in people with stroke: Case report
}

\author{
Amanda E. Chisholm, PhD; ${ }^{*}$ Taha Qaiser; Tania Lam, PT, PhD \\ School of Kinesiology, University of British Columbia, Vancouver, Canada; and International Collaboration on Repair \\ Discoveries, Vancouver Coastal Health Research Institute, Vancouver, Canada
}

\begin{abstract}
People are required to adapt their basic walking pattern to turn and change directions safely for activities of daily living. This case study describes the changes in neuromuscular control among individuals with stroke on walking paths of different curvatures. Two men with hemiparetic stroke and one control subject walked along a straight, wide curved, and tight curved pathway while muscle activation of the medial and lateral gastrocnemius was recorded, along with the trajectory of the center of pressure (COP) during the single support phase. Balance, sensorimotor control, and functional ambulation were also evaluated. The subject with greater lower-limb sensorimotor impairment displayed a larger difference in the anterior-posterior COP displacement between limbs, which exacerbated as the path curvature increased. In addition, while the control subject demonstrated a lateral shift in the mediallateral COP position, this person was unable to adapt the COP position on the nonparetic side. The second participant with a stroke demonstrated better balance and lateral shifting of the COP position. Modulation of the COP trajectory is required to safely perform curved walking. Further study is required to confirm the role of stroke-related gait deficits in the ability to adapt to curved walking.
\end{abstract}

Key words: biomechanics, center of pressure, curved walking, electromyography, gait, hemiparesis, kinetics, neuromuscular control, stroke, turning.

\section{INTRODUCTION}

Current approaches to gait assessment following stroke primarily measure impairments during straight walking as opposed to skilled tasks that require the ability to adapt the basic walking pattern. Recent studies have postulated that impairments in gait that persist after stroke, such as slow speed and spatial-temporal asymmetry [1-2], may limit the ability to adapt the gait pattern as required to mobilize safely in the community [3]. Turning and curved walking create a unique set of demands on the neural processes involved in the control of mediallateral (ML) stability and forward progression that are greater than the requirements for straight walking [4-8]. Given the significance of turning in everyday mobility [9-10], as well as the higher risk of falls and injuries while turning [11-15], it is surprising that there has been very little research to date on the capacity to adapt straight walking in ambulatory individuals with stroke.

Some clinical assessments of mobility, the Timed "Up and Go" test (TUG) and Emory Functional Ambulation Profile, include a turning task to examine adaptation to straight walking. Both measures have been correlated

Abbreviations: $\mathrm{AP}=$ anterior-posterior, $\mathrm{CMSA}=$ ChedokeMcMaster Stroke Assessment, COP = center of pressure, EMG = electromyography, FSR = force-sensitive resistor, LG = lateral gastrocnemius, $\mathrm{MG}=$ medial gastrocnemius, Mini-BESTest = Mini-Balance Evaluations Systems Test, ML = medial-lateral, TUG = Timed "Up and Go" test.

*Address all correspondence to Amanda E. Chisholm, PhD; International Collaboration on Repair Discoveries, 818 West 10th Ave, Vancouver, British Columbia, Canada, V5Z 1M9; 604-675-8815. Email: achisholm@icord.org http://dx.doi.org/10.1682/JRRD.2014.08.0189 
with balance function [16-17], walking speed [17-18], and lower-limb motor impairment [19-20] in the stroke population. Previous findings have demonstrated that individuals with chronic stroke require a greater number of steps and more time to complete a turn to both the paretic and nonparetic sides than controls [21]. Furthermore, turning capacity at $180^{\circ}$ was correlated with single-support time asymmetry, suggesting greater difficulty for individuals with more severe gait impairments [21]. Similarly, performance on the Figure-8 Walk Test shows that individuals with chronic stroke take twice as long to complete a curved walking task as nondisabled controls [22]. Although timed tests of functional ambulation offer an easy and efficient measure of gait performance in the clinical setting, they do not expose underlying challenges in the motor control of changing directions during walking that can be determined by examining the center of pressure (COP) trajectory.

Curved walking imposes greater ML instability than straight walking [4-7] because the COP needs to shift toward the lateral edge of the inner foot $[7,23]$. People with stroke have impairments in the regulation of frontal plane stability, as indicated by a reduction in weight-shifting capacity while standing [24-26]. However, the extent to which ML control is affected during curved walking remains unknown. Examining the COP trajectory may provide information on the capacity to turn or walk along curved paths. Individuals with stroke demonstrate less ML variability and shorter anterior-posterior (AP) displacement in the COP trajectory under the paretic foot than the nonparetic foot during straight walking [27]. These findings suggest poor control of ML stability and forward progression, which coincides with a reduced single support time on the paretic side [27]. In addition, this may lead to difficulty adapting the gait pattern to match the greater demands required of curved walking.

Modulation of muscle activation patterns has been identified with walking along curved pathways [28-29]. The inner leg of the turn shows reduced amplitude of activity in muscles located in the medial compartments of the lower limb, whereas those in the lateral compartments show increased amplitude with increasing path curvature; however, the opposite effect is observed for the outer leg [28]. These effects are consistent with a shift in foot pressures toward the lateral aspect of the inner foot [29]. One study investigating neuromuscular strategies for curved walking in people with chronic stroke found reduced adaptation in the gastrocnemii and hamstring muscles and a shift in foot pressures to increased curved walking paths compared with age-matched controls [29]. In addition, physiological evidence shows that the gastrocnemii muscles contribute to active control over ML ankle motion to stabilize the body in the frontal plane and may be related to the degree of challenge to stability [30]. While difficulties in curved walking are apparent among people with chronic stroke, examining the COP excursion can reveal aspects about the neuromuscular response to control ML stability and forward progression.

The purpose of this case report was to better understand neuromuscular control in walking along paths with increasing curvatures by examining changes in the COP trajectory and lower-limb muscle activity among people with stroke. We hypothesized that individuals with stroke would show impaired control over the ML stability and reduced forward progression as path curvature increased compared with reference data provided by an agematched control.

\section{METHODS}

\section{Case Description}

Two men who had an ischemic stroke that resulted in hemiparesis participated in this study after giving written informed consent. Subject 1 (S1) was 59 yr old and had a stroke 7 yr ago affecting his left side, while subject 2 (S2) was $61 \mathrm{yr}$ old with the right side affected from a stroke $1 \mathrm{yr}$ ago. Both participants were able to walk a minimum of 10 $\mathrm{m}$ without an assistive device. However, S1 uses a single point cane and ankle-foot orthotic for daily mobility. Neither of the subjects had experienced a fall since their stroke. Additionally, a $65 \mathrm{yr}$ old man, free of any other musculoskeletal or neurological disease affecting balance or mobility, completed the study as a control participant. All demographic and clinical data are outlined in Table 1. The University of British Columbia Clinical Research Ethics Board approved the experimental procedures.

\section{Measurements}

We evaluated the degree of sensorimotor impairment due to stroke with the Chedoke-McMaster Stroke Assessment (CMSA) impairment inventory for the foot and leg administered by a trained research assistant. Each item was measured on a 7-point scale, with lower scores indicating greater impairment. The CMSA has high interrater and intrarater reliability [31]. The Mini-Balance Evaluations 
Table 1.

Subject demographic and clinical data.

\begin{tabular}{lccc}
\hline \multicolumn{1}{c}{ Demographic } & Subject 1 & Subject 2 & Control \\
\hline Age (yr) & 59 & 61 & 65 \\
Weight (kg) & 81.6 & 70.3 & 79.3 \\
Height (cm) & 175 & 172 & 177 \\
Stroke Type & Ischemic & Ischemic & - \\
Time Poststroke (yr) & 7 & 1 & - \\
Affected Side & Left & Right & - \\
Falls & 0 & 0 & 0 \\
TUG + Dual Task (s) & 36.1 & 11.1 & 8.9 \\
Mini-BESTest & 13 & 27 & 28 \\
CMSA Leg & 5 & 6 & - \\
CMSA Foot & 2 & 5 & - \\
CMSA = Chedoke-McMaster Stroke Assessment (measure of sensorimotor \\
impairment), Mini-BESTest = Mini-Balance Evaluations Systems Test (mea- \\
sure of balance function), TUG = Timed “Up and Go” test (measure of func- \\
tional mobility).
\end{tabular}

Systems Test (Mini-BESTest) was used to assess balance control. The 14 items are scored from 0 (severe impairment) to 2 (normal function) to represent anticipatory balance, reactive postural control, sensory orientation, and dynamic gait functions. Interrater reliability is above 0.96 for the total score on the Mini-BESTest [32]. The TUG is an item of the Mini-BESTest and was used to assess functional mobility [32].

Two force-sensitive resistors (FSRs) (Interlink Electronics Inc; Camarillo, California) were positioned under the heel and medial forefoot of each foot to determine stance and swing phases of the gait cycle. Surface electromyography (EMG) (Delsys Inc; Boston, Massachusetts) was used to record activity of the medial gastrocnemius (MG) and lateral gastrocnemius (LG) bilaterally during walking. Subjects walked over a force plate positioned in the middle of the walkway (Bertec; Columbus, Ohio). FSR, EMG, and force plate data were collected at $1,000 \mathrm{~Hz}$ in a custom data acquisition program made in LabVIEW (National Instruments; Austin, Texas). Infrared-emitting markers (Optotrak, NDI; Waterloo, Canada) were used to track the motion of each foot, which were defined with the following landmarks: head of the fifth metatarsal, head of the second metatarsal, and heel. Kinematic data were synchronized with a trigger to the acquisition system and sampled at $100 \mathrm{~Hz}$. The kinematic landmarks were used to define the foot's position with respect to the force plate coordinate system.

\section{Experimental Protocol}

Data were recorded while participants traversed each of the following paths, which were outlined on the laboratory floor with tape (Figure 1):

1. Straight path: $6 \mathrm{~m}$ straight path, curvature $=0$.

2. Large semicircle: $10 \mathrm{~m}$ circular path with a radius of $2 \mathrm{~m}$, curvature $=0.5 \mathrm{~m}$.

3. Small semicircle: $3 \mathrm{~m}$ circular path with a radius of $0.5 \mathrm{~m}$, curvature $=2 \mathrm{~m}$.

Subjects walked clockwise and counterclockwise along each path at their usual comfortable walking speed without any assistive device until four to five acceptable foot contacts on the force plate were achieved per direction. Participants were monitored to ensure that they were following the path, such that the line outlining the path curvature was maintained between the two feet. The time required to complete each of these paths was recorded with a stopwatch to calculate walking speed. Sufficient rest was allowed between walking trials to minimize fatigue.

\section{Data Analysis}

Data were analyzed offline using custom-written routines in MATLAB (MathWorks; Natick, Massachusetts). FSR and force plate signals were low-pass filtered at $25 \mathrm{~Hz}$ with a fourth-order Butterworth filter. The EMG data were full-wave rectified and low-pass filtered at $25 \mathrm{~Hz}$ with a fourth-order Butterworth filter. FSRs data were used to define the times of foot contact and toe-off and identify the single support phase. The step cycle was defined as the period between successive foot contact

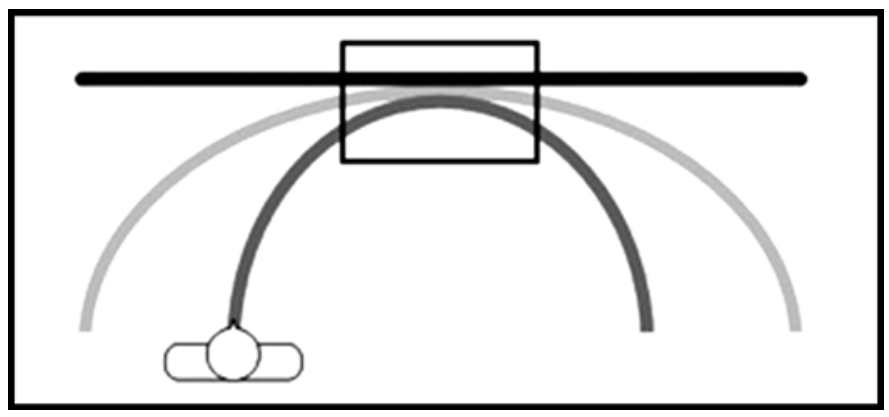

Figure 1.

Laboratory setup for straight (black line), wide curve (light gray line), and tight curve (dark gray line) walking paths were outlined with tape on floor. Force plate was positioned in middle of each path. 
times, and data were normalized to 100 percent of the gait cycle. Walking trials with insufficient foot contact on the force plate were excluded from the analysis. Data were divided to examine COP excursions and muscle activity during the single support phase for the inner leg when walking along the curve. Kinematic landmarks on the foot were used to normalize calculations for the AP COP displacement from the first to last COP position in the single support phase with respect to foot length and ML mean position and variability (standard deviation). These measures provided information on forward progression and ML stability during the single support phase. We also calculated the ML-COP minimum and maximum perpendicular distance relative to the lateral border of the foot (i.e., defined as a line from the heel to fifth metatarsal) to examine stability relative to the base of support limits. COP outcome measures were calculated for each trial and presented as the mean for each condition. The EMG data were normalized as a percentage of the gait cycle for each step over the force plate, and then the average amplitude was calculated over the gait cycle for each condition. Muscle activity from the single support phase of the step cycle was inspected to evaluate changes across conditions.

\section{RESULTS}

Table 2 presents a summary of the COP outcome measures. All subjects reduced their comfortable walking speed as the path curvature increased. The control participant demonstrated a reduction in AP-COP displacement as the path curvature increased. The ML-COP shows a trend toward greater variability and more lateral mean position during the tight curve compared with the wide curve and straight path (Figure 2, Table 2). As the path curvature increased, the ML minimum and maximum distance to the foot's lateral border increased. The MG activity decreased during single support as the path curvature increased, while LG activity shows the opposite trend (Figure 3).

Table 2.

Center of pressure outcome measures for straight and curved walking.

\begin{tabular}{|c|c|c|c|c|c|c|}
\hline \multirow{2}{*}{ Test Path } & \multirow{2}{*}{ Speed $(\mathrm{m} / \mathrm{s})$} & \multicolumn{4}{|c|}{ ML } & \multirow{2}{*}{$\begin{array}{c}\text { AP } \\
\text { Displacement }\end{array}$} \\
\hline & & Mean & Variability & Minimum & Maximum & \\
\hline \multicolumn{7}{|l|}{ Subject 1} \\
\hline \multicolumn{7}{|l|}{ Paretic } \\
\hline Wide & $0.36 \pm 0.08$ & $12.6 \pm 2.3$ & $1.5 \pm 0.2$ & $67.4 \pm 5.8$ & $76.8 \pm 3.8$ & $-8.7 \pm 3.3$ \\
\hline Tight & $0.30 \pm 0.01$ & $10.0 \pm 0.5$ & $1.5 \pm 0.5$ & $63.1 \pm 3.1$ & $70.3 \pm 3.4$ & $-6.0 \pm 5.1$ \\
\hline Wide & $0.40 \pm 0.05$ & $25.9 \pm 1.4$ & $2.8 \pm 1.2$ & $75.5 \pm 9.0$ & $101.7 \pm 4.1$ & $19.8 \pm 7.5$ \\
\hline Tight & $0.32 \pm 0.04$ & $28.2 \pm 3.5$ & $3.2 \pm 1.7$ & $72.1 \pm 4.2$ & $101.0 \pm 7.2$ & $19.3 \pm 4.2$ \\
\hline \multicolumn{7}{|l|}{ Subject 2} \\
\hline \multicolumn{7}{|l|}{ Paretic } \\
\hline Straight & $0.92 \pm 0.14$ & $8.0 \pm 2.8$ & $4.5 \pm 2.7$ & $35.4 \pm 10.2$ & $56.4 \pm 4.8$ & $49.7 \pm 21.8$ \\
\hline Wide & $0.89 \pm 0.17$ & $5.2 \pm 8.2$ & $5.9 \pm 2.3$ & $26.2 \pm 3.9$ & $67.9 \pm 7.8$ & $47.6 \pm 6.4$ \\
\hline Tight & $0.69 \pm 0.06$ & $7.1 \pm 4.0$ & $5.9 \pm 5.6$ & $48.5 \pm 7.6$ & $67.6 \pm 2.5$ & $29.6 \pm 12.7$ \\
\hline \multicolumn{7}{|l|}{ Control } \\
\hline Straight & $1.25 \pm 0.10$ & $7.1 \pm 3.9$ & $4.4 \pm 1.7$ & $29.2 \pm 7.4$ & $83.3 \pm 3.2$ & $58.0 \pm 1.8$ \\
\hline Wide & $0.99 \pm 0.04$ & $7.7 \pm 3.5$ & $4.3 \pm 1.4$ & $38.2 \pm 5.0$ & $89.2 \pm 1.2$ & $49.9 \pm 3.5$ \\
\hline Tight & $0.97 \pm 0.02$ & $13.4 \pm 4.7$ & $4.8 \pm 1.9$ & $41.1 \pm 6.9$ & $94.6 \pm 6.0$ & $44.9 \pm 7.0$ \\
\hline
\end{tabular}


(a)

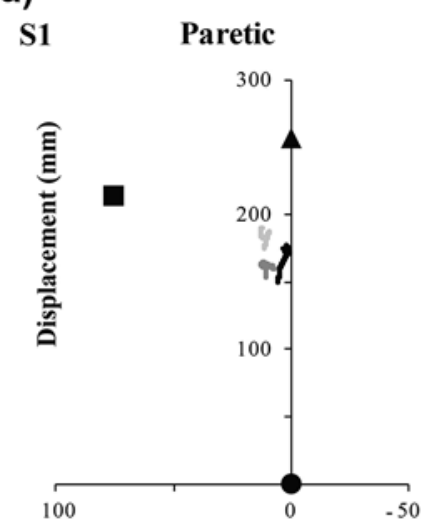

(b)

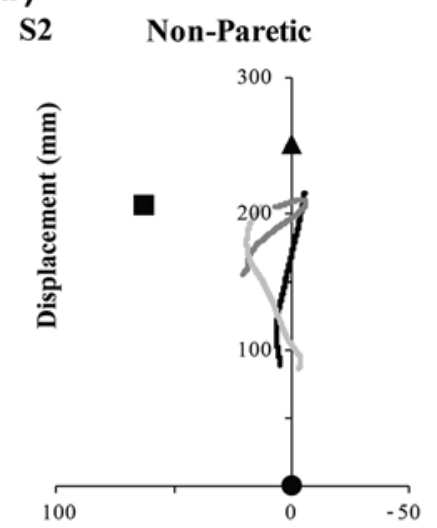

(c)

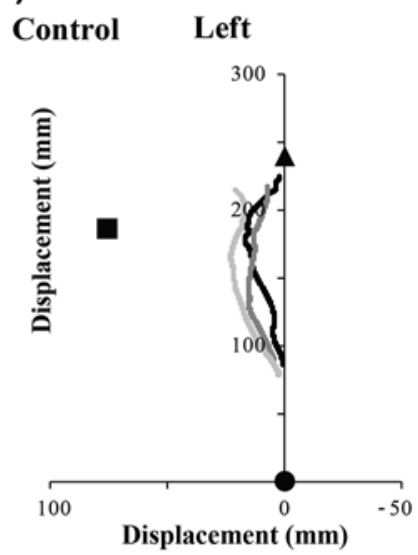

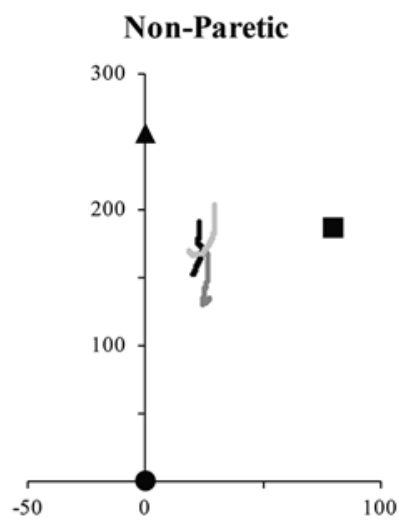

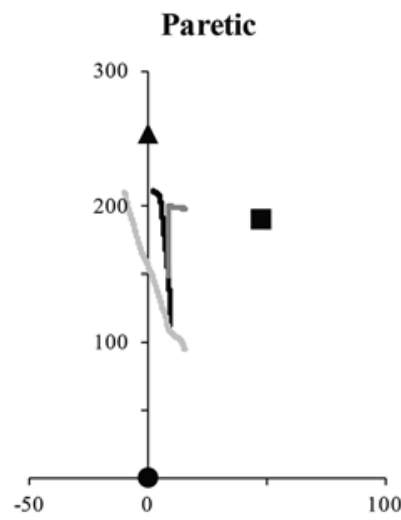

(a)

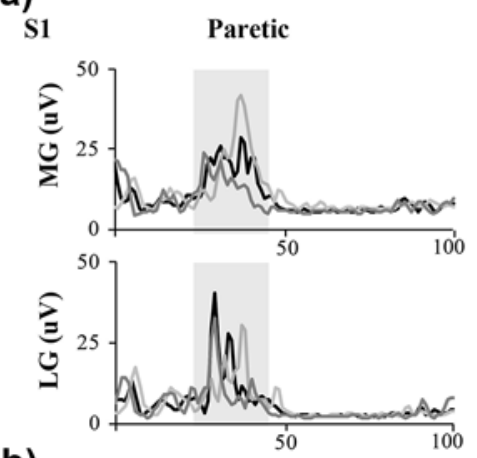

(b)
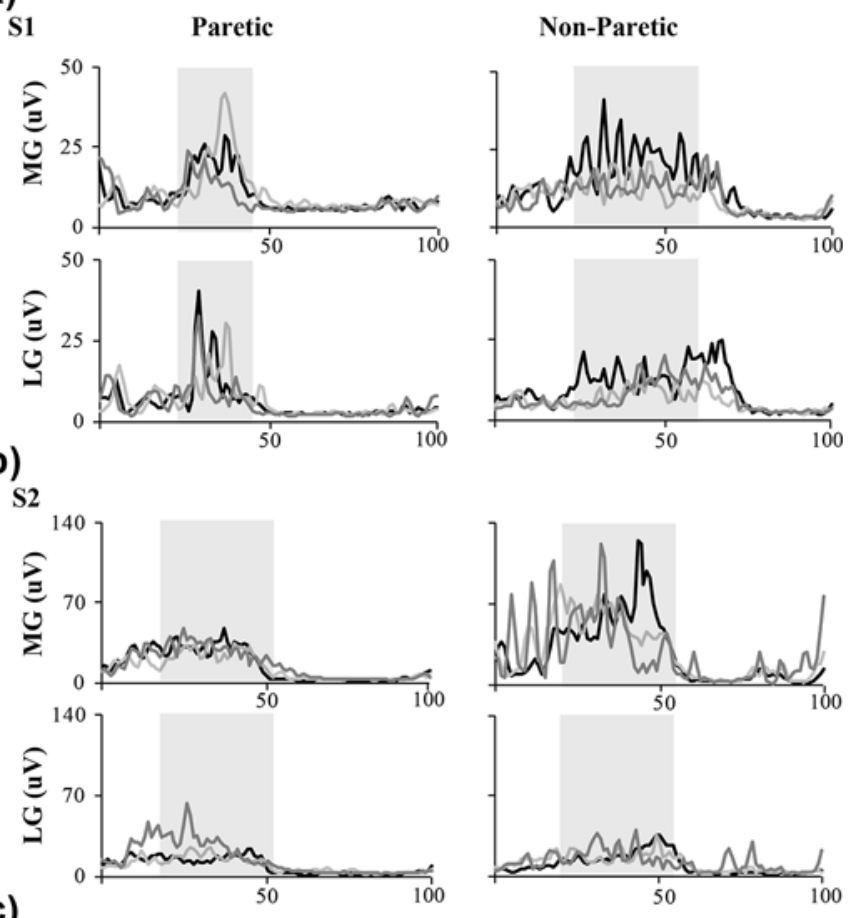

(c)
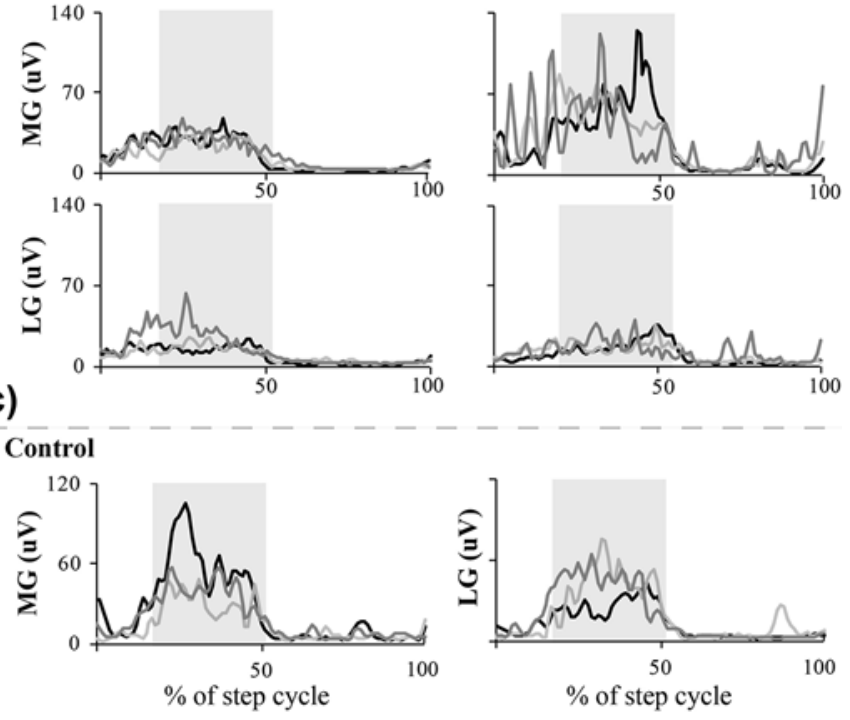

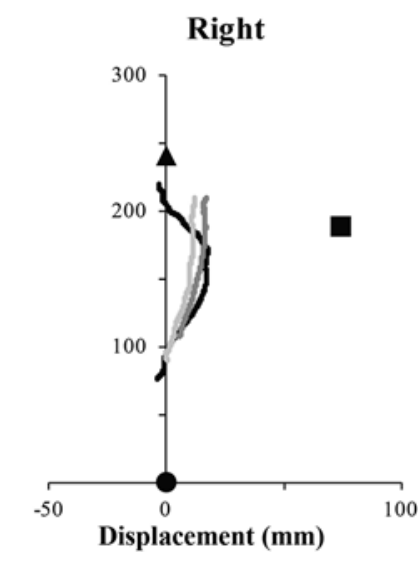

Right

Figure 2.

Each panel shows representative data of center of pressure trajectory, measured in millimeters, during straight (black line), wide curve (light gray line), and tight curve (dark gray line) walking paths for (a) S1, (b) S2, and (c) control participant. Positions of heel (black circle), second metatarsal (black triangle), and fifth metatarsal (black square) are plotted. $\mathrm{S}=$ subject.

S1 presented with severe lower-limb sensorimotor impairment and poor balance function. COP analysis

Figure 3.

Average electromyography activities for medial gastrocnemius (MG) and lateral gastrocnemius (LG) muscles are plotted for straight (black line), wide curve (light gray line), and tight curve (dark gray line) walking paths for (a) S1, (b) S2, and (c) control participant. Data are normalized to $100 \%$ of step cycle. Shaded gray box represents average single support phase. Note $y$-axis has been adjusted for subjects based on their maximum activity during step cycle. $\mathrm{S}$ = subject.

revealed a negative AP displacement for the paretic limb across all conditions for S1, indicating that the first COP point was anterior to the last COP point on the foot during the single support phase. The difference between the minimum and maximum point in the AP direction reveals mean displacements of $12.9,6.5$, and 4.4 percent (relative to foot length) for straight, wide curve, and tight curve paths, respectively. The paretic and nonparetic limbs show no change in ML variability between conditions. The nonparetic limb's AP displacement reduced as 
the path curvature increased; however, the ML variability was consistently larger for all conditions compared with the paretic limb (Table 2). The minimum and maximum ML-COP distance does not show the increasing trend across condition as observed in the control participant. MG and LG muscle activity show no change for both limbs as the path curvature increases (Figure 3).

In comparison to S1, S2 had mild lower-limb sensorimotor impairment, better balance function, and a faster walking speed. The AP-COP displacement for both limbs reduced as the path curvature increased. ML variability was greater during the curved conditions than in the straight condition for both limbs (Table 2). The ML minimum distance increased from the straight to tight path curvature for the nonparetic limb. The nonparetic limb showed no change in LG activity and a slight decrease in MG activity, while the paretic showed a slight increase in LG activity and no change in MG activity from straight to tight curved walking paths (Figure 3).

\section{DISCUSSION}

In this case report, we examined modulation of the COP trajectory and lower-limb muscle activity during the single support phase of gait in response to walking paths with increasing curvature among individuals with chronic stroke and an age-matched control participant. Muscular adaptations to curved walking, but not the COP trajectory, have been examined in nondisabled adults and people with stroke [28-29]. We observed reduction in the AP-COP displacement and an increase in ML variability as the walking path curvature increased, possibly reflecting the degree of difficulty in controlling frontal plane stability. However, the individual with stroke presenting with greater lower-limb sensorimotor impairment displayed poor forward progression on the paretic side and did not demonstrate modulation in ML variability with increasing curvatures on the nonparetic side. Other changes we detected include reduced walking speed, larger minimum ML-COP distance to the foot's lateral border, and a more lateral mean COP position.

Research on measuring the COP trajectory to understand neuromuscular control during walking in the stroke population has mainly focused on straight walking and gait initiation [27,33]. In comparison to straight paths, the COP trajectory is modulated during curved walking, as seen by the reduction in AP displacement, lateral shift in position, and greater ML variability. Similar to a previous study, S1 demonstrated poor forward progression on the paretic side and a greater difference between limbs for APCOP displacement than S2, likely due to a greater severity in lower-limb sensorimotor control [27]. The difference between limbs for AP-COP displacement may be related to challenges in controlling stability during forward progression over the paretic limb. Factors contributing to a smaller AP-COP displacement on the paretic side observed for S1 include a flatfoot or forefoot initial contact, reduced stance time, and poor stability [1,34]. Furthermore, the difference in AP-COP displacement between limbs appears to be further exacerbated as the path curvature increases in both subjects. Given that between-limb asymmetry is required to maintain curved walking [7], perhaps the lateral weight shift to the paretic side becomes more difficult as the curvature increases. In a previous study, individuals with greater temporal asymmetry took a longer time and more steps to complete a $180^{\circ}$ turn to both sides [21]. Individuals with gait asymmetry may require more specific training on curved walking and turning to improve lateral weight shifting capacity.

Challenges to frontal plane stability during curved walking are observed by changes in COP trajectory and ML mean position and variability. A more lateral ML position under the foot as the path curvature increased may reflect a strategy to maintain balance as the body adjusts to change directions. These findings are consistent with observations that people lean toward the inside leg as the pelvis shifts over to the inside of the curve $[5,28]$ and plantar pressures redistribute under that lateral aspect of the foot [29]. Greater ML variability may reflect an increased demand on lateral stability when challenged to adapt the basic locomotor pattern. In particular for S1, increased time on the nonparetic limb to compensate for poor swing phase mechanics of the paretic limb may contribute to a larger ML-COP variability. Individuals with greater sensorimotor impairment may engage compensatory movements, such as hip hiking and lower-limb circumduction, to facilitate swinging the paretic limb forward [35]. Therefore, these compensations may also contribute to increased ML-COP variability.

In agreement with previous studies, our control participant demonstrated a reduction in MG activity and an increase in LG activity to adapt to increases in the path curvature [28-29]. Modulation in muscle activity is thought to be necessary for maintaining ML stability during curved walking [4,7]. A previous study linked reduced 
modulation of the MG to increases in path curvature when the paretic limb was on the inside of the curve [29]. Our new observations on the muscular response show reduced MG activity and no change in LG activity on the nonparetic side as the path curvature increased. It has been suggested that cutaneous afferents may play a role in modulating EMG activity through the sural nerve reflex [36]. The lack of change in EMG activity on the nonparetic side for S1 may reflect no changes in ML-COP position as the curvature increased, thus leading to reduced activity of cutaneous afferents on the lateral aspect of the foot. Further study is required to confirm a relationship between modulation of cutaneous afferents, EMG activity, and COP trajectory.

Although we have described important changes in the neuromuscular control response to curved walking, there are several limitations to be considered. Our participants walked at their comfortable pace for each condition, which reduced as the path curvature increased. Slower walking speeds have been demonstrated to produce a shorter AP-COP displacement and smaller ML$\mathrm{COP}$ variability in the stroke population [27]. Future controlled studies will require a matched speed across conditions to discern the effects of walking speed and curve difficulty. While we examined adaptation to curved walking without the influence of an assistive device (e.g., cane or walker), these devices are commonly prescribed to maintain walking capacity to compensate for underlying impairments following a stroke [37]. Although previous studies have suggested that assistive devices can alter the gait pattern and the degree of symmetry between limbs [38-39], the effect of assistive devices on curved walking has yet to be investigated. This will be important to provide better application of research findings to the walking conditions encountered in daily activities.

Our findings provide an initial report showing difficulty in adapting the neuromuscular response to curved walking among people with stroke. Although we initially collected data from eight individuals poststroke, only data from two individuals were included in the analysis because of an insufficient number of acceptable foot contacts on the force plate during curved walking $(<4$ per condition). For future studies conducting this type of assessment, we recommend limiting the number of walking conditions collected to avoid fatigue and using multiple force plates to capture more foot contacts per walking bout. In addition, we only focused on the inner leg of the turn because the body shifts toward the inner leg during curved walking [7]. However, future studies should also explore contributions of the outer leg to the motor control of curved walking. In addition, it will be important to establish the optimal amount of change in the outcome measures between walking conditions. Our findings also highlight characteristics of individuals who may be suitable to complete this assessment, such as ability to walk $10 \mathrm{~m}$ without an assistive device, mild sensorimotor impairment at the leg (CMSA 5+), and a normal rating on the walking items of the Mini-BESTest. This will guide future studies with a larger sample to confirm the effect of walking curvature on neuromuscular control.

\section{CONCLUSIONS}

This case report describes our initial findings on modulation of the COP trajectory and muscle activity in response to curved walking. Our results add to research showing that after stroke individuals have difficulty adapting walking conditions that involve curves or turns $[21,29,40]$. We found a reduction in the AP-COP displacement and an increase in ML variability as the walking path curvature increased, which may indicate the degree of difficulty in maintaining stability. Developing new approaches to evaluate walking capacity is critical to determining the effect of interventions on the underlying neuromuscular control for walking tasks performed in everyday activities. Further studies are required to understand changes in COP trajectory and muscle activity with respect to stroke-related impairments.

\section{ACKNOWLEDGMENTS}

\section{Author Contributions:}

Study concept and design: T. Lam.

Acquisition of data: A. E. Chisholm, T. Qaiser.

Analysis and interpretation of data: A. E. Chisholm, T. Qaiser, T. Lam.

Drafting of manuscript: A. E. Chisholm.

Critical revision of manuscript for important intellectual content:

A. E. Chisholm, T. Qaiser, T. Lam.

Final approval for manuscript to be published: A. E. Chisholm, T. Qaiser, T. Lam.

Financial Disclosures: The authors have declared that no competing interests exist.

Funding/Support: This material was based on work supported by the Canadian Institutes of Health Research (reference number 93375).

Institutional Review: The University of British Columbia Clinical Research Ethics Board approved the experimental procedures. 
Participant Follow-Up: The authors do not plan to inform participants of the publication of this study. However, participants have been encouraged to check the Web site for updated publications.

\section{REFERENCES}

1. Olney SJ, Richards C. Hemiparetic gait following stroke. Part I: Characteristics. Gait Posture. 1996;4:136-48. http://dx.doi.org/10.1016/0966-6362(96)01063-6

2. Patterson KK, Parafianowicz I, Danells CJ, Closson V, Verrier MC, Staines WR, Black SE, McIlroy WE. Gait asymmetry in community-ambulating stroke survivors. Arch Phys Med Rehabil. 2008;89(2):304-10. [PMID:18226655] http://dx.doi.org/10.1016/j.apmr.2007.08.142

3. Roerdink M, Lamoth CJ, Kwakkel G, van Wieringen PC, Beek PJ. Gait coordination after stroke: Benefits of acoustically paced treadmill walking. Phys Ther. 2007;87(8): 1009-22. [PMID:17553922] http://dx.doi.org/10.2522/ptj.20050394

4. Courtine G, Schieppati M. Human walking along a curved path. I. Body trajectory, segment orientation and the effect of vision. Eur J Neurosci. 2003;18(1):177-90.

[PMID:12859351]

http://dx.doi.org/10.1046/j.1460-9568.2003.02736.x

5. Patla AE, Adkin A, Ballard T. Online steering: Coordination and control of body center of mass, head and body reorientation. Exp Brain Res. 1999;129(4):629-34.

[PMID:10638436]

http://dx.doi.org/10.1007/s002210050932

6. Imai T, Moore ST, Raphan T, Cohen B. Interaction of the body, head, and eyes during walking and turning. Exp Brain Res. 2001;136(1):1-18. [PMID:11204402] http://dx.doi.org/10.1007/s002210000533

7. Orendurff MS, Segal AD, Berge JS, Flick KC, Spanier D, Klute GK. The kinematics and kinetics of turning: Limb asymmetries associated with walking a circular path. Gait Posture. 2006;23(1):106-11. [PMID:16311202] http://dx.doi.org/10.1016/j.gaitpost.2004.12.008

8. Courtine G, Schieppati M. Human walking along a curved path. II. Gait features and EMG patterns. Eur J Neurosci. 2003;18(1):191-205. [PMID:12859352] http://dx.doi.org/10.1046/j.1460-9568.2003.02737.x

9. Glaister BC, Bernatz GC, Klute GK, Orendurff MS. Video task analysis of turning during activities of daily living. Gait Posture. 2007;25(2):289-94. [PMID:16730441] http://dx.doi.org/10.1016/j.gaitpost.2006.04.003

10. Sedgman R, Goldie P. Development of a measure of turning during walking. Proceedings of the Inaugural Conference of Faculty of Health Sciences, La Trobe University; 1994; Melbourne, Australia.
11. Hyndman D, Ashburn A, Stack E. Fall events among people with stroke living in the community: Circumstances of falls and characteristics of fallers. Arch Phys Med Rehabil. 2002;83(2):165-70. [PMID:11833018] http://dx.doi.org/10.1053/apmr.2002.28030

12. Jørgensen L, Engstad T, Jacobsen BK. Higher incidence of falls in long-term stroke survivors than in population controls: Depressive symptoms predict falls after stroke. Stroke. 2002;33(2):542-47. [PMID:11823667] http://dx.doi.org/10.1161/hs0202.102375

13. Harris JE, Eng JJ, Marigold DS, Tokuno CD, Louis CL. Relationship of balance and mobility to fall incidence in people with chronic stroke. Phys Ther. 2005;85(2):150-58. [PMID:15679466]

14. Nevitt MC, Cummings SR, Hudes ES. Risk factors for injurious falls: A prospective study. J Gerontol. 1991;46(5): M164-70. [PMID:1890282] http://dx.doi.org/10.1093/geronj/46.5.M164

15. Cumming RG, Klineberg RJ. Fall frequency and characteristics and the risk of hip fractures. J Am Geriatr Soc. 1994; 42(7):774-78. [PMID:8014355] http://dx.doi.org/10.1111/j.1532-5415.1994.tb06540.x

16. Knorr S, Brouwer B, Garland SJ. Validity of the Community Balance and Mobility Scale in community-dwelling persons after stroke. Arch Phys Med Rehabil. 2010;91(6): 890-96. [PMID:20510980] http://dx.doi.org/10.1016/j.apmr.2010.02.010

17. Wolf SL, Catlin PA, Gage K, Gurucharri K, Robertson R, Stephen K. Establishing the reliability and validity of measurements of walking time using the Emory Functional Ambulation Profile. Phys Ther. 1999;79(12):1122-33. [PMID:10630281]

18. van Hedel HJ; EMSCI Study Group. Gait speed in relation to categories of functional ambulation after spinal cord injury. Neurorehabil Neural Repair. 2009;23(4):343-50.

[PMID:19036717] http://dx.doi.org/10.1177/1545968308324224

19. Manaf H, Justine M, Omar M. Functional balance and motor impairment correlations with gait parameters during timed up and go test across three attentional loading conditions in stroke survivors. Stroke Res Treat. 2014;2014: 439304. [PMID:24757575] http://dx.doi.org/10.1155/2014/439304

20. Baer HR, Wolf SL. Modified Emory Functional Ambulation Profile: An outcome measure for the rehabilitation of poststroke gait dysfunction. Stroke. 2001;32(4):973-79. [PMID:11283399] http://dx.doi.org/10.1161/01.STR.32.4.973

21. Lam T, Luttmann K. Turning capacity in ambulatory individuals poststroke. Am J Phys Med Rehabil, 2009;88(11): 873-83; quiz 884-86, 946. [PMID:19893383] 
22. Wong SS, Yam MS, Ng SS. The Figure-of-Eight Walk test: Reliability and associations with stroke-specific impairments. Disabil Rehabil. 2013;35(22):1896-1902. [PMID:23600714] http://dx.doi.org/10.3109/09638288.2013.766274

23. Hase K, Stein RB. Turning strategies during human walking. J Neurophysiol. 1999;81(6):2914-22. [PMID:10368408]

24. Pai YC, Rogers MW, Hedman LD, Hanke TA. Alterations in weight-transfer capabilities in adults with hemiparesis. Phys Ther. 1994;74(7):647-57, discussion 657-59. [PMID:8016197]

25. Turnbull GI, Charteris J, Wall JC. Deficiencies in standing weight shifts by ambulant hemiplegic subjects. Arch Phys Med Rehabil. 1996;77(4):356-62. [PMID:8607759] http://dx.doi.org/10.1016/S0003-9993(96)90084-2

26. Dettmann MA, Linder MT, Sepic SB. Relationships among walking performance, postural stability, and functional assessments of the hemiplegic patient. Am J Phys Med. 1987; 66(2):77-90. [PMID:3578493]

27. Chisholm AE, Perry SD, McIlroy WE. Inter-limb centre of pressure symmetry during gait among stroke survivors. Gait Posture. 2011;33(2):238-43. [PMID:21167716]

http://dx.doi.org/10.1016/j.gaitpost.2010.11.012

28. Courtine G, Papaxanthis C, Schieppati M. Coordinated modulation of locomotor muscle synergies constructs straight-ahead and curvilinear walking in humans. Exp Brain Res. 2006;170(3):320-35. [PMID:16328271] http://dx.doi.org/10.1007/s00221-005-0215-7

29. Duval K, Luttin K, Lam T. Neuromuscular strategies in the paretic leg during curved walking in individuals poststroke. J Neurophysiol. 2011;106(1):280-90.

[PMID:21562197]

http://dx.doi.org/10.1152/jn.00657.2010

30. Vieira TM, Minetto MA, Hodson-Tole EF, Botter A. How much does the human medial gastrocnemius muscle contribute to ankle torques outside the sagittal plane? Hum Mov Sci. 2013;32(4):753-67. [PMID:23992638]

http://dx.doi.org/10.1016/j.humov.2013.03.003

31. Gowland C, Stratford P, Ward M, Moreland J, Torresin W, Van Hullenaar S, Sanford J, Barreca S, Vanspall B, Plews N. Measuring physical impairment and disability with the ChedokeMcMaster Stroke Assessment. Stroke. 1993;24(1):58-63.

[PMID:8418551] http://dx.doi.org/10.1161/01.STR.24.1.58

32. Tsang CS, Liao LR, Chung RC, Pang MY. Psychometric properties of the Mini-Balance Evaluation Systems Test (Mini-BESTest) in community-dwelling individuals with chronic stroke. Phys Ther. 2013;93(8):1102-15.

[PMID:23559522]

http://dx.doi.org/10.2522/ptj.20120454
33. Hesse S, Reiter F, Jahnke M, Dawson M, Sarkodie-Gyan T, Mauritz KH. Asymmetry of gait initiation in hemiparetic stroke subjects. Arch Phys Med Rehabil. 1997;78(7):719-24. [PMID:9228874] http://dx.doi.org/10.1016/S0003-9993(97)90079-4

34. Gaviria M, D’Angeli M, Chavet P, Pelissier J, Peruchon E, Rabischong P. Plantar dynamics of hemiplegic gait: A methodological approach. Gait Posture. 1996;4:297-305. http://dx.doi.org/10.1016/0966-6362(95)01055-6

35. Chen G, Patten C, Kothari DH, Zajac FE. Gait differences between individuals with post-stroke hemiparesis and nondisabled controls at matched speeds. Gait Posture. 2005; 22(1):51-56. [PMID:15996592] http://dx.doi.org/10.1016/j.gaitpost.2004.06.009

36. Zehr EP, Stein RB, Komiyama T. Function of sural nerve reflexes during human walking. J Physiol. 1998;507(Pt 1): 305-14. [PMID:9490858] http://dx.doi.org/10.1111/j.1469-7793.1998.305bu.x

37. Laufer Y. The use of walking aids in the rehabilitation of stroke patients. Rev Clin Gerontol. 2004;14(2):137-44. http://dx.doi.org/10.1017/S0959259805001449

38. Beauchamp MK, Skrela M, Southmayd D, Trick J, Kessel MV, Brunton K, Inness E, McIlroy WE. Immediate effects of cane use on gait symmetry in individuals with subacute stroke. Physiother Can. 2009;61(3):154-60.

[PMID:20514177] http://dx.doi.org/10.3138/physio.61.3.154

39. Tyson SF. Hemiplegic gait symmetry and walking aids. Physiother Theory Pract. 1994;10(3):153-59. http://dx.doi.org/10.3109/09593989409036392

40. Faria CD, Teixeira-Salmela LF, Nadeau S. Effects of the direction of turning on the timed up \& go test with stroke subjects. Top Stroke Rehabil. 2009;16(3):196-206. [PMID:19632964] http://dx.doi.org/10.1310/tsr1603-196

Submitted for publication August 14, 2014. Accepted in revised form June 1, 2015.

This article and any supplementary material should be cited as follows:

Chisholm AE, Qaiser T, Lam T. Neuromuscular control of curved walking in people with stroke: Case report. J Rehabil Res Dev. 2015;52(7):775-84. http://dx.doi.org/10.1682/JRRD.2014.08.0189

ResearcherID: Tania Lam, PT, PhD: H-3730-2015

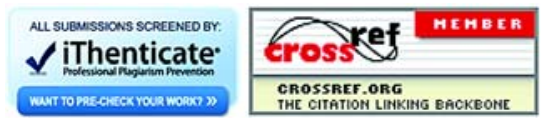


